\title{
О РЕЧЕМЫСЛИТЕЛЬНЫХ ЗАДАЧАХ В ОБУЧЕНИИ МАТЕМАТИКЕ В УСЛОВИЯХ БИЛИНГВИЗМА
}

\author{
Туктамышов Н.К. ${ }^{1}$
}

${ }^{1}$ ФБОУ ВПО «Казанский государственный архитектурно-строительный университет», Казань, е-таіl: info@kgasu.ru

Республика Татарстан (Российская Федерация) является многоязычной. По данным Министерства образования в Республике Татарстан около 1256 школ с обучением на татарском языке, а в 410 школах обучение ведется на билингвальной основе (татарско-русский, чувашско-русский, мари-русский и т.д.). Целью данной работы является исследование потенциала речемыслительных задач в условиях билингвального обучения при подготовке инженера-строителя. С опорой на принцип единства языка и мышления выясняются причины разобшенности мышления и речи на втором языке и способы их объединения на базе создания комплекса учебных речемыслительных задач. В работе приведен фрагмент занятия, посвященного обыкновенным дифференциальным уравнениям. Как показали эксперименты, наибольшие трудности у студентов возникают при выполнении таких мыслительных операций, как абстрагирование, анализ и синтез. Обучение математике в условиях билигвизма путем решения речемыслительных задач позволяет проанализировать и сравнить математический контент в различных языках; повысить культуру математической речи на русском и татарском языках; улучшить речевые и мыслительные механизмы, логическую память, преодолеть разрывы в родном и втором языке; повысить культуру математической речи на русском и татарском языках; улучшить речевые и мыслительные механизмы, логическую память, преодолеть разрывы в родном и втором языках; усилить когнитивный интерес, формировать математические и языковые компетенции на втором языке.

Ключевые слова: мышление, речь, язык, математика, билингвальное обучение

\section{ABOUT INTELLECTUAL-SPEECH TASKS IN TEACHING MATHEMATICS IN CONDITION OF BILINGUALISM}

\author{
Tuktamyshov N.K. ${ }^{1}$
}

${ }^{1}$ Kazan State University of Architecture and Engineering, Kazan, e-mail: info@kgasu.ru

Republic of Tatarstan (Russian Federation) is multilingual. According to the Ministry of Education of the Republic of Tatarstan, there are about 1256 schools with teaching in the Tatar language, and 410 schools, where teaching is conducted on a bilingual basis (Tatar-Russian, Chuvash-Russian, Mari-Russian, etc.). The aim of this work is to study the potential of intellectual-speech tasks against the background of bilingual teaching in professional training of a civil engineer. The causes of segregation between thinking and speech in the second language can be clarified relying on the principle of unity of language and thinking, as well as the ways for their unification by means creating a scheme of educational intellectual-speech tasks. The paper shows a fragment of a class dedicated to ordinary differential equations. As shown by experiments, students experience the difficulties when performing such mental operations as abstraction, analysis and synthesis. Teaching mathematics against the background of bilingualism, using methods of solving intellectual-speech tasks makes it possible to: analyze and compare mathematical content in different languages; improve the culture of mathematical speech in Russian and Tatar languages; improve speech and cognitive mechanisms, logical memory; fill gaps in both the native and the second language; strengthen cognitive interest and form math and language competencies in a second language.

Keywords: thinking, speech, language, mathematics, bilingual teaching

Если в 1990 г. татар, обучающихся на родном языке, было около 24\%, то к 2012 г. этот показатель достигает 47\% (данные Министерства образования Республики Татарстан). В некоторых вузах Республики Татарстан обучение ведется на билингвальной основе (русскотатарской - на основе русского, татарско-русской - на основе татарского). Как правило, обучение на двуязычной основе ведется на младших курсах, что позволяет студентам 
адаптироваться к дальнейшему обучению на русском языке.

Проблема взаимоотношения мышления и речи очень сложна [1-3]. Л.С. Выготский [1, c. 380-381] описывал этот как процесс: «...от мотива, порождающего какую-либо мысль, к оформлению самой мысли, к опосредованию ее во внутреннем слове, затем в значениях внешних слов и, наконец, в словах». В данной работе при обучении математике на билингвальной основе предлагается использовать приемы решения речемыслительных задач.

Теоретическую базу нашего исследования составляют работы Б.В. Гнеденко [4], А.Я. Хинчина [5], В. Barton [6], посвященные изучению роли языка в обучении математике.

В работах Л.Л. Салеховой, Н.К. Туктамышова [7] предлагается методика, использующая речемыслительные задачи и направленная на развитие математической речи студентов. При этом под речемыслительной деятельностью понимается единый процесс порождения мысли и речи, который составляет материальную базу общения: слушание, сознательное усвоение нового учебного материала, говорение, чтение и письмо.

Цель исследования. Выяснение причины разобщенности мышления и речи на втором языке и выявление потенциала речемыслительных задач в обучении математике на билингвальной основе в вузах Республики Татарстан.

Обучение академической дисциплине на втором языке предполагает одномоментную концентрацию обучающегося не только на контенте, но и на форме изложения. Обычно, обучаясь на втором языке, студенты переводят или интерпретируют неизвестный концепт на свой родной, но в таком случае отсутствует реальный процесс обобщения явлений объективной реальности. Возникает разъединение речи и мышления на втором языке. Устранение указанного несоответствия можно осуществить путем составления специальных заданий в виде речемыслительных задач, в ходе решения которых мыслительная деятельность учащегося направлена на реальный предмет мысли, а не на иноязычные языковые средства выражения мысли. Мыслительная деятельность основана на отражательной способности человека и осуществляет познавательную функцию, в то время как решение речемыслительных задач предстает в качестве разновидности первой, но с «функцией» как бы вторичного познания. В ходе решения речемыслительных задач мыслительная деятельность направлена на неязыковой предмет; речь отрабатывается на умственных действиях; достигается автоматизм речевого действия; умственные и речевые действия поддаются контролю со стороны преподавателя.

Материалы и методы исследования. Среди современных исследований вопросов и проблем мышления имеются работы, в которых говорится о национально-культурных особенностях мыслительного процесса посредством понятий и кодов $[8,9]$. В связи со сказанным следует отметить, что порядок слов в предложениях на русском и татарском 
языке различается. Главное отличие заключается в том, что глагол в татарском предложении всегда пишется в его конце. Имеется существенное различие, например, в произношении дробей на русском и татарском языках. В русском языке сначала произносится значение числителя, а потом знаменателя, а в татарском - наоборот. В ходе обучения на билингвальной основе необходимо применение методики, опирающейся на знания студентов, полученные в монолингвальном режиме, предполагающем формирование математического понятийного аппарата на втором языке как процесс узнавания уже известных представлений и отношений. При этом, поскольку нет изоморфизма между языками, то особое значение при введении нового понятия приобретает подведение под него готового понятия или определения, учитывающего семантическое поле слова, являющегося фокусом речевого высказывания. Семантическое поле подразумевает многомерную систему смысловых связей данного слова с другими лексическими единицами языка, а акт номинации предмета существует в форме индивидуально развивающегося речемыслительного процесса. Значение слова в акте называния равносильно той операции, с помощью которой мыслится предмет, то есть пониманию интеллектуальных операций со значениями слов. Осознание семантического поля на родном языке ведет к его осознанию на русском языке.

В ходе обучения математике на двуязычной основе находят применение речемыслительные задачи разного типа. Л.Л. Салехова [10] речемыслительные задачи условно разделяет на понятийно-лексические, чисто математические и ситуативномыслительные. Понятийно-лексическая задача предоставляет новый математический концепт на втором языке, позволяющий достигать понимания учащимися без перевода. Чисто математические задачи позволяют запрограммировать на конкретной лексике определенное количество мыслительных операций и проследить за каждым шагом студента. Таким образом, они уводят его мыслительную деятельность от иноязычной языковой формы, направляя ее на реальный предмет мысли.

Приведем для иллюстрации применения речемыслительных задач, используя понятийно-лексические и чисто математические задачи, фрагмент занятия, посвященного обыкновенным дифференциальным уравнениям. Занятие разбивается условно на две части. Первая часть посвящена решению с помощью преподавателя понятийно-лексических задач на втором языке, что предполагает освоение новых понятий.

Часть 1. Преподаватель объявляет тему у доски и объясняет материал.

Обыкновенным дифференциальным уравнением первого порядка называется уравнение вида:

$$
\mathrm{F}\left(\mathrm{x}, \mathrm{y}, \mathrm{y}^{\prime}\right)=0 .(1)
$$


Здесь F - заданная непрерывная функция своих аргументов, х - независимая переменная, у - искомая функция, $\mathrm{y}^{\prime}$ - производная от искомой функции. Напомним, что $\mathrm{y}^{\prime}=\mathrm{dy} / \mathrm{dx}$, отсюда $\mathrm{dy}=\mathrm{y}^{\prime} \mathrm{dx}$ (эта формула записывается на доске). Обыкновенное дифференциальное уравнение (так как здесь не рассматриваются дифференциальные уравнения в частных производных, то в дальнейшем будем говорить «дифференциальное уравнение») (1) можно записать в виде (записывается на доске)

$$
\mathbf{y}^{\prime}=\mathbf{f}(\mathbf{x}, \mathbf{y}),(2)
$$

где $\mathrm{f}$ - известная непрерывная функция своих аргументов. Порядок уравнения равен порядку наивысшей производной уравнения (1).

Решением дифференциального уравнения (1) или (2) называется всякая функция $\mathrm{y}=\varphi(\mathrm{x})$, которая, будучи подставлена в уравнение (1) или (2), обратит его в тождество.

Общее решение дифференциального уравнения первого порядка дается формулой $\mathrm{y}=\varphi(\mathrm{x}, \mathrm{C})$. Здесь $\varphi-$ известная функция, имеющая одну произвольную постоянную С. Это предварительное, неточное определение, но вполне пригодное для наших целей (сообщается устно).

Вторая часть имеет в виду решение математических задач, использующих новую лексику первой части. Преподаватель на практическом занятии объявляет тему у доски и объясняет материал.

Уравнения с разделенными переменными. (Преподаватель пишет на доске тему занятия)

Указанные уравнения имеют вид

$\mathrm{dy} / \mathrm{dx}=-\mathrm{M}(\mathrm{x}) / \mathrm{N}(\mathrm{y})$ или вытекающий отсюда эквивалентный вид

$$
\mathrm{M}(\mathrm{x}) \mathrm{dx}+\mathrm{N}(\mathrm{y}) \mathrm{dy}=0 \text {, }
$$

где M, N соответственно заданные непрерывные функции только от х, у. Решение такого уравнения получается прямым интегрированием

$\int \mathrm{M}(\mathrm{x}) \mathrm{dx}+\int \mathrm{N}(\mathrm{y}) \mathrm{dy}=\mathrm{C}$, где $\mathrm{C}-$ произвольная постоянная.

Рассмотрим дифференциальное уравнение с разделяющимися переменными.

Уравнения с разделяющимися переменными. Такие уравнения имеют вид

$y^{\prime}=-M(x) N(y) / M^{\prime}(x) N^{\prime}(y)$

или равносильный вид

$\mathrm{M}(\mathrm{x}) \mathrm{N}(\mathrm{y}) \mathrm{dx}+\mathrm{M}^{\prime}(\mathrm{x}) \mathrm{N}^{\prime}(\mathrm{y}) \mathrm{dy}=0,(5)$

где $M(x), N(y), M^{\prime}(x), N^{\prime}(x)$ - известные функции. Для решения такого уравнения нужно проделать ряд шагов.

Первый шаг. Освобождаемся от дробей, если они есть (вид (4)).

Второй шаг. Соединяем в один все члены, содержащие один и тот же дифференциал. 
Уравнение примет вид (5).

Третий шаг. Далее дифференциальное уравнение приведем к виду (3).

Четвертый шаг. Получаем решение.

Пример. Далее преподаватель решает пример совместно со студентами. На доске преподаватель записывает уравнение.

$\mathrm{dy} / \mathrm{dx}=\left(1+\mathrm{y}^{2}\right) /\left(\left(1+\mathrm{x}^{2}\right) \mathrm{xy}\right)$.

Преподаватель: является ли данное уравнение дифференциальным?

Студент 1: Да, поскольку оно содержит производную. Это обыкновенное дифференциильное уравнение первого порядка.

Первый шаг. Преподаватель просит студента 1 выполнить первый шаг

$\left(1+x^{2}\right) x y d y=\left(1+y^{2}\right) d x$

Второй шаг. Преподаватель: Является ли уравнение уравнением с разделенными переменными?

Студент 1: Является, кажется?

Студент 2: Не является, так как перед дифференцииалами записаны выражения, зависящие как от $x$, так и от $y$.

Преподаватель: Студент 2 прав, и это ключевой момент, поскольку переменные еще не разделень;; студент 2, попробуйте разделить переменные в уравнении

$\left(1+\mathrm{y}^{2}\right) \mathrm{dx}-\mathrm{x}\left(1+\mathrm{x}^{2}\right) \mathrm{ydy}=0$.

Студент 2 (после некоторого обдумывания): Чтобы отделить переменные, нужно перейти к третьему шагу, а для этого поделим обе части уравнения на $x\left(1+x^{2}\right)\left(1+y^{2}\right)$. Это daem

$d x /\left(\left(x\left(1+x^{2}\right)\right)-y d y /\left(1+y^{2}\right)=0\right.$.

Преподаватель: Хорошо. Далее перейдем к четвертому шагу.

Проводится краткая работа по припоминанию соответствующих формул, и интегралы вычисляются. В итоге

$\operatorname{Ln}\left(1+x^{2}\right)\left(1+y^{2}\right)=2 \ln x-2 C$.

Преподаватель: Результат можно написать в более сжатом виде

$\left(1+x^{2}\right)\left(1+y^{2}\right)=C x^{2}-$ это общий интеграл уравнения.

Основные положения речи педагога записываются студентами. Таким образом, в результате изложения данной темы студентами достигнуто беспереводное понимание следующих понятий и терминов: «обыкновенное дифференциальное уравнение», «общее решение дифференциального уравнения», «уравнение с разделенными (разделяющимися) переменными», «общий интеграл» и т.д.

Анализ показывает, что наиболее важными для решения задачи являются 
мыслительные операции второго и четвертого шагов. Можно исходя из этого за решение задачи проставить баллы.

5 баллов.

1. Умение представить задачу в виде дифференциального соотношения, разглядеть вид (класс) дифференциального уравнения

$\mathrm{M}(\mathrm{x}) \mathrm{N}(\mathrm{y}) \mathrm{dx}+\mathrm{M}^{\prime}(\mathrm{x}) \mathrm{N}^{\prime}(\mathrm{y}) \mathrm{dy}=0$.

2. Преобразование уравнения, разделение переменных, приведение уравнения к стандартному виду.

3. Представление решения в виде алгебраической суммы интегралов.

4. Преобразование подынтегральных выражений, приведение их к табличным интегралам:

a) при интегрировании не потерять произвольную постоянную и не приобрести вместо одной две произвольные постоянные;

б) приведение общего решения к простейшему виду.

4 балла. Студент может выполнить пункты 1-4, но допускает в пункте 4 ошибки в подпунктах а) и б).

3 балла. Студент определяет тип уравнения, удовлетворяет пунктам 1-3, но не может найти первообразные интегралов.

2 балла. Может выполнить пункты $1-2$.

1 балл. Выполняет пункт 1.

0 баллов. Студент не может выполнить ни одного из пунктов 1-4.

Результаты исследования и их обсуждение. Приведем таблицу 1, отражающую алгоритм решения задачи, а также мыслительные и математические операции.

Таблица 1

Показатели мыслительных и математических операций

\begin{tabular}{|c|c|c|}
\hline Шаг & Мыслительные операции & Математические операции \\
\hline$y^{\prime}=\frac{M(x) N(y)}{M^{\prime}(x) N^{\prime}(Y)}$ & $\begin{array}{l}\text { Анализ и синтез } \\
\text { (преобразование, включенное } \\
\text { в первый шаг, предполагает } \\
\begin{array}{l}\text { анализ } \\
\text { приведение } \\
\text { стандартному виду) }\end{array}\end{array}$ & $\begin{array}{l}\quad \text { Освобождение } \\
\text { знаменателя элементарными } \\
\text { алгебраическими } \\
\text { преобразованиями } \\
M^{\prime}(x) N^{\prime}(y) d y=-M(x) N(y) d x\end{array}$ \\
\hline 2 & \begin{tabular}{l}
\multicolumn{3}{c}{ Сравнение, } \\
классификация, \\
распределение по п. \\
группам.
\end{tabular} & $\begin{array}{c}\text { Перенос } \\
\text { уравнения в левую часть } \\
M^{\prime}(x) N^{\prime}(y) d y+M(x) N(y) d x=0\end{array}$ \\
\hline
\end{tabular}




\begin{tabular}{|c|c|c|}
\hline $\begin{array}{l}M^{\prime}(x) N^{\prime}(y) d y= \\
=-M(x) N(y) d x\end{array}$ & $\begin{array}{l}\text { Здесь уравнение отнесли к } \\
\text { классу уравнений } \\
\text { разделяющимися } \\
\text { переменными }\end{array}$ & \\
\hline $\begin{array}{l}M^{\prime}(x) N^{\prime}(y) d y+ \\
+M(x) N(y) d x=0\end{array}$ & \begin{tabular}{lr}
\multicolumn{1}{c}{ Анализ } & и \\
конкретизация, \\
устанавливаются \\
пвязи \\
возмольку \\
между \\
алгебраического объекта (связи
\end{tabular} & $\begin{array}{l}\text { Деление обеих частей } \\
\text { уравнения на соответствующее } \\
\text { выражение } \\
\qquad \frac{M(x)}{M^{\prime}(x)} d x+\frac{N^{\prime}(y)}{N(y)} d y=0\end{array}$ \\
\hline $\begin{array}{l}\frac{M(x)}{M^{\prime}(x)} d x+ \\
+\frac{N^{\prime}(y)}{N(y)} d y=0\end{array}$ & $\begin{array}{l}\text { Анализ и синтез, } \\
\text { сравнение, абстрагирование, } \\
\text { сравнение, конкретизация }\end{array}$ & \begin{tabular}{l}
\multicolumn{2}{c}{ Получение } \\
решения уравнения \\
интегрирования обем \\
уравнения. \\
$\int \frac{M(x)}{M^{\prime}(x)} d x+\int \frac{N^{\prime}(y)}{N(y)} d y=$ const
\end{tabular} \\
\hline
\end{tabular}

В Казанском государственном архитектурно-строительном университете (КГАСУ) был проведен эксперимент. Давались 4 задачи для самостоятельного решения, аналогичные приведенным ранее. Участники - студенты первого курса Института строительства КГАСУ, поступившие в университет в 2016 г. Число участников 31. В таблице 2 приведены результаты эксперимента.

Таблица 2

Оценка мыслительных операций

\begin{tabular}{|l|l|l|l|l|l|}
\hline \multicolumn{1}{|c|}{ Баллы } & 5 & 4 & 3 & 2 & 1 \\
\hline $\begin{array}{l}\text { Количество } \\
\text { студентов }\end{array}$ & 7 & 9 & 13 & 2 & 0 \\
\hline
\end{tabular}

Как показали эксперименты, наибольшие трудности у студентов возникают на четвертом шаге, т.е. они испытывают затруднения при выполнении таких мыслительных операций, как абстрагирование, анализ и синтез. В результате решения понятийнолексических задач первой части проблемы, связанные с лексикой, в основном снимаются. Только 2 из 31 студента не до конца поняли как постановку задачи, так и из-за плохого овладения объясненными ранее понятиями и терминами - смысл решения дифференциального уравнения. Затруднения у оставшихся студентов не носят понятийно- 
лексический характер и совпадают с затруднениями, имеющими место и у студентов, обучающихся на родном языке. Это позволяет в какой-то мере (требующей тем не менее еще дополнительных экспериментов) говорить о том, что студенты мыслят на втором языке.

Заключение. Таким образом, обучение математике в условиях билигвизма путем решения речемыслительных задач позволяет:

- проанализировать и сравнить математический контент в различных языках;

- повысить культуру математической речи на русском и татарском языках;

- улучшить речевые и мыслительные механизмы, логическую память, преодолеть разрывы в родном и втором языках;

- усилить когнитивный интерес и формировать математические и языковые компетенции на втором языке.

\section{Список литературы}

1. Выготский Л.С. Мышление и речь. М.: Лабиринт, 1999. 350 с.

2. Глухов В.П. Психолингвистика. М.: В. Секачёв. 2014. 343 с.

3. Леонтьев А.А. Слово в речевой деятельности: Некоторые проблемы общей теории речевой деятельности. M.: URSS. 2014. 256 с.

4. Гнеденко Б.В. Воспоминания. Моя жизнь в математике и математика в моей жизни. М.: Либроком, 2012. 624 с.

5. Хинчин А.Я. Восемь лекций по математическому анализу. М.: УРСС. 2015. 280 с.

6. Barton B. The language of mathematics. Telling mathematical tales. N.Y.: Springer, 2009. $186 \mathrm{p}$.

7. Salekhova L.L., Tuktamyshov N.K. Bilingual mathematics teaching in conditions of higher educational establishment. Proceedings of the ICMI study 21 conference: mathematics education and language diversity. Aguas de Lindoia. Sao Paolo state. Brazil. 2011. P. 341-346.

8. Габдулхаков В.Ф., Хисамова В.Н. Лингводидактика поликультурного образования. М.: Национальный книжный центр. 2012. 248 с.

9. Верещагин В.М., Костомаров В.Г. Язык и культура. Москва-Берлин: Директ-медиа, 2014. $532 \mathrm{c}$.

10. Салехова Л.Л. Дидактическая модель билингвального обучения математике в высшей педагогической школе: дис. ...доктора пед. наук. Казань, 2008. 428 с. 